\title{
Microvascular Invasion as a Prognostic Predictor in Hepatocellular Carcinoma: How Accurate Is It?
}

\author{
Teh-la Huo ${ }^{a-c}$ Shu-Yein Hob, d, e Chia-Yang Hsu ${ }^{f}$ \\ ${ }^{a}$ Department of Medical Research, Taipei Veterans General Hospital, Taipei, Taiwan; \\ ${ }^{b}$ Faculty of Medicine, National Yang-Ming University School of Medicine, Taipei, Taiwan; \\ CInstitute of Pharmacology, National Yang-Ming University School of Medicine, \\ Taipei, Taiwan; ${ }^{\mathrm{d}}$ Department of Medicine, Taipei Veterans General Hospital, Taipei, Taiwan; \\ e Division of Gastroenterology and Hepatology, Min-Sheng General Hospital, \\ Taoyuan, Taiwan; fDivision of Gastroenterology and Hepatology, University of \\ Michigan, Ann Arbor, MI, USA
}

Dear Editor

We read the paper "Microvascular Invasion and a Size Cutoff Value of $2 \mathrm{~cm}$ Predict LongTerm Oncological Outcome in Multiple Hepatocellular Carcinoma: Reappraisal of the American Joint Committee on Cancer Staging System and Validation Using the Surveillance, Epidemiology, and End-Results Database" by Dr. Shindoh et al., published in a recent issue of Liver Cancer [1], with enormous interest. Two large surgical cohorts (1,175 patients in the training set and 5,249 patients in the validation set) were analyzed to investigate the prognostic significance of microvascular invasion (MVI) and its ability to improve the performance of AJCC system in hepatocellular carcinoma (HCC). Although their findings are interesting, a few concerns may deserve further discussion.

Independent studies indicated that MVI was a major risk factor for tumor recurrence in patients with HCC undergoing surgical treatment [2,3]. Although MVI is an important prognostic predictor for HCC, the confirmation of MVI can only be demonstrated from the serially slice-cut resected specimen postoperatively. This is especially crucial when the prevalence rate of MVI was reported to reach $40.5 \%$ for tumors $\leq 2 \mathrm{~cm}$ and $49.6 \%$ for tumors $2.1-4 \mathrm{~cm}$ in diameter, respectively, in a surgical series of 322 patients [2]. Notably, the reported prevalence rate of MVI in Shindoh et al.'s series [1] could be profoundly underestimated (only $25.8 \%$ for median tumor diameter of $2.2 \mathrm{~cm}$ in the training cohort and $26.8 \%$ for median tumor diameter of $5.0 \mathrm{~cm}$ in the validation cohort). Underestimation of MVI could significantly compromise its prognostic ability in terms of outcome prediction. Consistent with this speculation is that in Shindoh et al.'s series [1], a new staging model based on MVI had only a 
slightly better performance than the AJCC 8th edition in both the training cohort (c-statistics, 0.648 vs. 0.629 ) and the validation cohort (c-statistics, 0.646 vs. 0.645 ) with marginal prediction benefits.

The potentially high frequency of MVI in small HCC is further supported by a classical study that looked at the explant livers in HCC patients undergoing liver transplantation [4]. Tiny satellite nodules or tumor nests $(<0.5 \mathrm{~cm})$ were frequently identified in the explants through serially examining the slice-cut specimen, thus greatly limiting the ability of imaging detection tools in these cases. In summary, MVI acts as a distinct tumor behavior and may serve as a unique prognostic marker for HCC. For patients who receive curative resection, identification of MVI should be best demonstrated from multiple, serially slice-cut resected pathological specimens. To avoid possible underestimation of MVI, a thorough examination of surgical specimen is mandatory and considered the standard way of confirmation. Accurate information on MVI is expected to play an important role in refining the currently used staging system for HCC.

\section{Conflict of Interest Statement}

The authors have no conflicts of interest to declare.

\section{Funding Sources}

This study was supported by a grant from Taipei Veterans General Hospital (VN109-07), Taiwan.

\section{Author Contributions}

Guarantor of the article: T.-I. Huo. Specific author contributions: T.-I. Huo and C.-Y. Hsu performed the research. T.-I. Huo and S.-Y. Ho designed the study and wrote the paper. All authors approved the final version of the manuscript.

\section{References}

1 Shindoh J, Kobayashi Y, Kawamura Y, Akuta N, Kobayashi M, Suzuki Y, et al. Microvascular invasion and a size cutoff value of $2 \mathrm{~cm}$ predict long-term oncological outcome in multiple hepatocellular carcinoma: reappraisal of the American Joint Committee on Cancer Staging System and validation using the surveillance, epidemiology, and end-results database. Liver Cancer. 2020 Apr;9(2):156-66.

2 Tsai TJ, Chau GY, Lui WY, Tsay SH, King KL, Loong CC, et al. Clinical significance of microscopic tumor venous invasion in patients with resectable hepatocellular carcinoma. Surgery. 2000 Jun;127(6):603-8.

3 Imamura H, Matsuyama Y, Tanaka E, Ohkubo T, Hasegawa K, Miyagawa S, et al. Risk factors contributing to early and late phase intrahepatic recurrence of hepatocellular carcinoma after hepatectomy. J Hepatol. 2003 Feb;38(2):200-7.

4 Rizzi PM, Kane PA, Ryder SD, Ramage JK, Gane E, Tan KC, et al. Accuracy of radiology in detection of hepatocellular carcinoma before liver transplantation. Gastroenterology. 1994 Nov;107(5):1425-9. 